\title{
Fractional derivatives of the generalized Mittag-Leffler functions
}

\author{
Denghao Pang ${ }^{1} \mathbb{D}$, Wei Jiang ${ }^{1 *}$ and Azmat U.K. Niazi
}

\author{
"Correspondence: \\ jiangwei@ahu.edu.cn \\ ${ }^{1}$ School of Mathematical Sciences, \\ Anhui University, Hefei, China
}

\begin{abstract}
In this paper, we derive the compositions of the fractional derivatives with the Shukla function, a four-parameter Mittag-Leffler function. We investigate and compare the difference between the Riemann-Liouville and Caputo derivatives of the generalized Mittag-Leffler functions and obtain the reason causing the difference and expand the fractional derivatives of the generalized Mittag-Leffler functions. Two illustrative examples and the related numerical results are provided to demonstrate the validity.
\end{abstract}

MSC: $33 \mathrm{E} 12 ; 26 \mathrm{~A} 33$

Keywords: Mittag-Leffler function; Riemann-Liouville derivative; Caputo derivative

\section{Introduction}

In 2007, Shukla and Prajapati [1] introduced a four-parameter Mittag-Leffler function $E_{\alpha, \beta}^{\rho, q}(z)$ defined as

$$
E_{\alpha, \beta}^{\rho, q}(z)=\sum_{k=0}^{\infty} \frac{(\rho)_{q k} z^{k}}{\Gamma(k \alpha+\beta) k !} \quad(\alpha, \beta, \rho \in \mathbb{C}, q \in(0,1) \cup \mathbb{N}),
$$

where $\operatorname{Re}(\alpha)>0, \operatorname{Re}(\rho)>0$, and $(\rho)_{q k}=\frac{\Gamma(\rho+q k)}{\Gamma(\rho)}$. In the paper, the authors presented its various integral transforms and connections with other special functions, but they did not provide any relations of fractional derivatives. Obviously, it is a generalization of the MittagLeffler function (MLF). Generally speaking, the exponential function $e^{z}$, the Mittag-Leffler function $E_{\alpha}(z)$, the Wiman function $E_{\alpha, \beta}(z)$, and the Prabhakar function $E_{\alpha, \beta}^{\rho}(z)$ are particular cases of the Shukla function $E_{\alpha, \beta}^{\rho, q}(z)$.

In recent decades, with successful employing the fractional calculus in modeling the physical abnormal phenomena, the generalized MLFs frequently appeared in mathematical and physical problems because they were naturally presented along with the solution of fractional integral and differential equations. Therefore the mathematicians paid more attention to the behavior of the MLFs and extended their results to the complex domain. Until now, many properties of the highlighted special function have been derived (see [133] and the references therein). The earliest appearance of the MLFs dates back to 1903 when Magnus Gösta Mittag-Leffler [2] introduced the classical MLF $E_{\alpha}(z)$ as a special

(c) The Author(s) 2018. This article is distributed under the terms of the Creative Commons Attribution 4.0 International License (http://creativecommons.org/licenses/by/4.0/), which permits unrestricted use, distribution, and reproduction in any medium, provided you give appropriate credit to the original author(s) and the source, provide a link to the Creative Commons license, and indicate if changes were made. 
function of the form

$$
E_{\alpha}(z)=\sum_{k=0}^{\infty} \frac{z^{k}}{\Gamma(k \alpha+1)} \quad(\alpha \in \mathbb{C}, \operatorname{Re}(\alpha)>0)
$$

Two years later, Wiman [3] defined the two-parameter MLF $E_{\alpha, \beta}(z)$ as

$$
E_{\alpha, \beta}(z)=\sum_{k=0}^{\infty} \frac{z^{k}}{\Gamma(k \alpha+\beta)} \quad(\alpha \in \mathbb{C}, \operatorname{Re}(\alpha)>0)
$$

which is a generalization of the MLF and is now called the Wiman function. The early and lengthy work focused on the essential properties of the MLFs as entire functions and remained on the theoretical level of pure mathematics. Three decades later, the MLFs' application period has finally arrived. In 1930, Hille and Tamarkin [4] applied them in solving the Abel integral equations. In 1947, Gross [5] utilized the MLFs to study the creep and relaxation functions. Barrett [6] in 1954 was the pioneer to present the solution for a fractional differential equation with the MLFs. In 1971, using them, Caputo and Mainardi [7] studied the fractional viscoelasticity. With the deep-going research, in 1971, Prabhakar [8] introduced another generalization of MLFs, a three-parameter MLF $E_{\alpha, \beta}^{\rho}(z)$, defined as the follows:

$$
E_{\alpha, \beta}^{\rho}(z)=\sum_{k=0}^{\infty} \frac{(\rho)_{k} z^{k}}{\Gamma(k \alpha+\beta) k !} \quad(\alpha, \beta, \rho \in \mathbb{C}, \operatorname{Re}(\alpha)>0),
$$

which is popular among fractional differential equations with three or more terms, where $(\rho)_{0}=1$ and $(\rho)_{k}=\frac{\Gamma(\rho+k)}{\Gamma(\rho)}$. In 1995, Kilbas and Saigo [9] generalized the MLF to another three-parameter one, containing a special entire function:

$$
E_{\alpha, m, l}(z)=\sum_{k=0}^{\infty} c_{k} z^{k} \quad(\alpha \in \mathbb{C}, m, l \in \mathbb{R}, \operatorname{Re}(\alpha)>0, m>0),
$$

where $c_{0}=1$ and $c_{k}=\prod_{i=0}^{k-1} \frac{\Gamma(\alpha(i m+l)+1)}{\Gamma(\alpha(i m+l+1)+1)}(k=1,2, \ldots)$. In recent years, the MLFs, together with the fractional calculus, have been applied in modeling the evolution of systems with memory ([17-33]), and many MATLAB routines with desired precision for evaluating the MLFs were given by I. Podlubny, Y.Q. Chen, and D.Y. Xue in the MATLAB Central.

Many results about the generalized MLFs have been presented; however, to our knowledge, there are few results about fractional derivatives of the Shukla function. Since the left Caputo derivative keeps the Mittag-Leffler function invariant, that is,

$$
\left({ }^{C} D_{a+}^{q} E_{q}\left(\lambda(z-a)^{q}\right)\right)(t)=\lambda E_{q}\left(\lambda(t-a)^{q}\right)
$$

it is interesting whether it does for the Shukla function. It is well known that $\left(\mathbb{D}_{a+1}^{\mu} 1\right)(t) \neq$ $\left({ }^{C} D_{a+}^{\mu} 1\right)(t)$ and $\left(\mathbb{D}_{a+}^{\mu} E_{\mu}\left(\lambda(t-a)^{\mu}\right)\right)(t) \neq\left({ }^{C} D_{a+}^{\mu} E_{\mu}\left(\lambda(t-a)^{\mu}\right)\right)(t)$, where $\mathbb{D}_{a+}^{\mu}$ is the left Riemann-Liouville fractional derivative, and ${ }^{C} D_{a+}^{\mu}$ is the left Caputo one. Our paper is devoted to the compositions of fractional derivatives with the Shukla function (1.1) and to the study of differences between the Riemann-Liouville (RL) and Caputo derivatives of the MLFs. The remainder of our paper is arranged as follows. Section 2 collects some 
basic definitions and results. In Sect. 3, we derive the fractional derivatives of the generalized MLFs. In the last section, we present examples to demonstrate the validity of our main results.

\section{Preliminaries}

This section collects some fundamental definitions and necessary lemmas.

Definition 2.1 ([14]) The left Riemann-Liouville fractional integral $\mathbb{I}_{a+}^{\mu}$ and derivative $\mathbb{D}_{a+}^{\mu}$ of order $\mu$ of a function $g(t) \in C[a, b]$ are defined as

$$
\left(\mathbb{I}_{a+}^{\mu} g\right)(t)=\frac{1}{\Gamma(\mu)} \int_{a}^{t}(t-\xi)^{\mu-1} g(\xi) d \xi \quad(\mu \in \mathbb{C}, \operatorname{Re}(\mu)>0)
$$

and

$$
\left(\mathbb{D}_{a+}^{\mu} g\right)(t)=\frac{1}{\Gamma(n-\mu)}\left(\frac{d}{d t}\right)^{n} \int_{a}^{t}(t-\xi)^{n-\mu-1} g(\xi) d \xi \quad(\mu \in \mathbb{C}, \operatorname{Re}(\mu)>0)
$$

where $n=[\operatorname{Re}(\mu)]+1$, and $[\operatorname{Re}(\mu)]$ denotes the integral part of $\operatorname{Re}(\mu)$.

Definition 2.2 ([14]) The left Caputo fractional derivative ${ }^{C} D_{a^{+}}^{\mu}$ of order $\mu$ of a function $g(t) \in C^{n}[a, b]$ is defined as

$$
{ }^{C} D_{a+}^{\mu} g(t)=\frac{1}{\Gamma(n-\mu)} \int_{0}^{t}(t-\xi)^{n-\mu-1} g^{(n)}(\xi) d \xi=\mathbb{I}_{a+}^{n-\mu} g^{(n)}(t),
$$

where $\mu \in \mathbb{C}$ and $\operatorname{Re}(\mu)>0$.

Lemma 2.3 ([14]) If $\mu, v \in \mathbb{C}, R(\mu) \geq 0$, and $R(v)>0$, then

$$
\left(\mathbb{I}_{a+}^{\mu}(s-a)^{\nu-1}\right)(t)=\frac{\Gamma(v)}{\Gamma(v+\mu)}(t-a)^{\nu+\mu-1} \quad(\operatorname{Re}(\mu)>0)
$$

and

$$
\left(\mathbb{D}_{a+}^{\mu}(s-a)^{\nu-1}\right)(t)=\frac{\Gamma(\nu)}{\Gamma(v-\mu)}(t-a)^{\nu-\mu-1} \quad(\operatorname{Re}(\mu) \geq 0) .
$$

In particular, if $v=1$ and $R(\mu) \geq 0$, then the Riemann-Liouville $(R L)$ derivative of a constant $C$ is

$$
\left(\mathbb{D}_{a+}^{\mu} C\right)(t)=\frac{C(t-a)^{-\mu}}{\Gamma(1-\mu)} \quad(0<\operatorname{Re}(\mu)<1) .
$$

Lemma 2.4 ([14]) Let $\mu, v \in \mathbb{C}, R(\mu) \leq 0$, and $R(v)>0$. Then, for $\operatorname{Re}(v)>n$, we have

$$
\left({ }^{C} D_{a+}^{\mu}(s-a)^{\nu-1}\right)(t)=\frac{\Gamma(v)}{\Gamma(v-\mu)}(t-a)^{\nu-\mu-1}
$$

and

$$
\left({ }^{C} D_{a+}^{\mu}(s-a)^{j}\right)(t)=0 \quad(j=0,1, \ldots, n-1) .
$$


In particular,

$$
\left({ }^{C} D_{a+}^{\mu} C\right)(t)=0 \quad(C \text { is a constant }) .
$$

Lemma 2.5 ([14]) Let $\mu, \lambda \in \mathbb{C}, \operatorname{Re}(\mu)>0$, and $a \in R$. Then

$$
\left(\mathbb{D}_{a+}^{\mu} E_{\mu}\left(\lambda(z-a)^{\mu}\right)\right)(t)=\frac{(t-a)^{-\mu}}{\Gamma(1-\mu)}+\lambda E_{\mu}\left(\lambda(t-a)^{\mu}\right) .
$$

Lemma 2.6 ([14]) Let $\mu, \lambda \in \mathbb{C}, \operatorname{Re}(\mu)>0$, and $a \in R$. Then

$$
\left({ }^{C} D_{a+}^{\mu} E_{\mu}\left(\lambda(z-a)^{\mu}\right)\right)(t)=\lambda E_{\mu}\left(\lambda(t-a)^{\mu}\right) .
$$

Lemma 2.7 ([16]) If $\alpha, \beta \in \mathbb{C}, \operatorname{Re}(\alpha)>0$, and $n \in \mathbb{N}$. Then

$$
z^{n} E_{\alpha, \beta+n \alpha}(z)=E_{\alpha, \beta}(z)-\sum_{k=0}^{n-1} \frac{z^{k}}{\Gamma(\beta+k \alpha)} .
$$

In particular,

$$
E_{\alpha, \beta}(z)=z E_{\alpha, \alpha+\beta}(z)+\frac{1}{\Gamma(\beta)} .
$$

\section{Main results}

In this section, we investigate the compositions of the fractional derivatives with the Shukla function (1.1). First of all, we know that it is an entire function [16]. The compositions are given as follows.

Theorem 3.1 Let $\mu, \alpha, \beta, \rho, \lambda \in \mathbb{C}, \operatorname{Re}(\mu)>0, \operatorname{Re}(\alpha)>0, \operatorname{Re}(\beta)>0, \operatorname{Re}(\rho)>0$, and $q \in \mathbb{N}$. Then

$$
\left(\mathbb{D}_{a+}^{\mu}(z-a)^{\beta-1} E_{\alpha, \beta}^{\rho, q}\left(\lambda(z-a)^{\alpha}\right)\right)(t)=(t-a)^{\beta-\mu-1} E_{\alpha, \beta-\mu}^{\rho, q}\left(\lambda(t-a)^{\alpha}\right) .
$$

Proof From (1.1) the left side of (3.1) can be changed o

$$
\left(\mathbb{D}_{a+}^{\mu}(z-a)^{\beta-1} E_{\alpha, \beta}^{\rho, q}\left(\lambda(z-a)^{\alpha}\right)\right)(t)=\left(\mathbb{D}_{a+}^{\mu} \sum_{k=0}^{\infty} \frac{(\rho)_{q k} \lambda^{k}(z-a)^{k \alpha+\beta-1}}{\Gamma(k \alpha+\beta) k !}\right)(t) .
$$

Because of the convergence, we can differentiate each individual term with (2.5):

$$
\begin{aligned}
\left(\mathbb{D}_{a+}^{\mu}(z-a)^{\beta-1} E_{\alpha, \beta}^{\rho, q}\left(\lambda(z-a)^{\alpha}\right)\right)(t) & =\sum_{k=0}^{\infty} \frac{(\rho)_{q k} \lambda^{k} \Gamma(k \alpha+\beta)(t-a)^{k \alpha+\beta-1-\mu}}{k ! \Gamma(k \alpha+\beta) \Gamma(k \alpha+\beta-\mu)} \\
& =\sum_{k=0}^{\infty} \frac{(\rho)_{q k} \lambda^{k}(t-a)^{k \alpha+\beta-1-\mu}}{k ! \Gamma(k \alpha+\beta-\mu)} \\
& =(t-a)^{\beta-\mu-1} E_{\alpha, \beta-\mu}^{\rho, q}\left(\lambda(t-a)^{\alpha}\right) .
\end{aligned}
$$


Remark 3.2 Formula (3.1) generalizes the RL derivative of Prabhakar's function

$$
\left(\mathbb{D}_{a+}^{\mu}(z-a)^{\beta-1} E_{\alpha, \beta}^{\rho}\left(\lambda(z-a)^{\alpha}\right)\right)(t)=(t-a)^{\beta-\mu-1} E_{\alpha, \beta-\mu}^{\rho}\left(\lambda(t-a)^{\alpha}\right),
$$

the RL derivative of Wiman's function

$$
\left(\mathbb{D}_{a+}^{\mu}(z-a)^{\beta-1} E_{\alpha, \beta}\left(\lambda(z-a)^{\alpha}\right)\right)(t)=(t-a)^{\beta-\mu-1} E_{\alpha, \beta-\mu}\left(\lambda(t-a)^{\alpha}\right),
$$

and Lemma (2.5). Substituting $\alpha=\mu$ and $\rho=q=\beta=1$ into (3.1) and applying (2.13), we obtain

$$
\begin{aligned}
\left(\mathbb{D}_{a+}^{\mu} E_{\mu}\left(\lambda(z-a)^{\mu}\right)\right)(t) & =(t-a)^{-\mu} E_{\mu, 1-\mu}\left(\lambda(t-a)^{\mu}\right) \\
& =\frac{(t-a)^{-\mu}}{\Gamma(1-\mu)}+\lambda E_{\mu}\left(\lambda(t-a)^{\mu}\right) .
\end{aligned}
$$

From Lemmas (2.5) and (2.6) we can find that the results are distinct. Let us check the Caputo derivative of Shukla's function (1.1).

Theorem 3.3 Let $\mu, \alpha, \beta, \rho, \lambda \in \mathbb{C}, \operatorname{Re}(\mu)>0, \operatorname{Re}(\alpha)>0, \operatorname{Re}(\beta)>0, \operatorname{Re}(\rho)>0$, and $q \in N$. Then, for $\operatorname{Re}(\beta) \neq 1$, we have

$$
\begin{aligned}
& \left({ }^{C} D_{a+}^{\mu}(z-a)^{\beta-1} E_{\alpha, \beta}^{\rho, q}\left(\lambda(z-a)^{\alpha}\right)\right)(t)=(t-a)^{\beta-\mu-1} E_{\alpha, \beta-\mu}^{\rho, q}\left(\lambda(t-a)^{\alpha}\right), \\
& \left({ }^{C} D_{a+}^{\mu}(z-a)^{\beta-1} E_{\alpha, \beta}^{\rho}\left(\lambda(z-a)^{\alpha}\right)\right)(t)=(t-a)^{\beta-\mu-1} E_{\alpha, \beta-\mu}^{\rho}\left(\lambda(t-a)^{\alpha}\right),
\end{aligned}
$$

and

$$
\left({ }^{C} D_{a+}^{\mu}(z-a)^{\beta-1} E_{\alpha, \beta}\left(\lambda(z-a)^{\alpha}\right)\right)(t)=(t-a)^{\beta-\mu-1} E_{\alpha, \beta-\mu}\left(\lambda(t-a)^{\alpha}\right) .
$$

In particular, for $\alpha=\mu$,

$$
\left({ }^{C} D_{a+}^{\mu}(z-a)^{\beta-1} E_{\mu, \beta}\left(\lambda(z-a)^{\mu}\right)\right)(t)=\lambda(t-a)^{\beta-1} E_{\mu, \beta}\left(\lambda(t-a)^{\mu}\right)+\frac{(t-a)^{\beta-\mu-1}}{\Gamma(\beta-\mu)} .
$$

Proof According to (1.1), we can rewrite the left side of (3.5) as

$$
\left({ }^{C} D_{a+}^{\mu}(z-a)^{\beta-1} E_{\alpha, \beta}^{\rho, q}\left(\lambda(z-a)^{\alpha}\right)\right)(t)=\left({ }^{C} D_{a+}^{\mu} \sum_{k=0}^{\infty} \frac{(\rho)_{q k} \lambda^{k}(z-a)^{k \alpha+\beta-1}}{\Gamma(k \alpha+\beta) k !}\right)(t) .
$$

Since the the series converges, we can interchange the order of the Caputo derivative and summation. Applying (2.7) straightway to (3.9), we derive

$$
\begin{aligned}
\left({ }^{C} D_{a+}^{\mu}(z-a)^{\beta-1} E_{\alpha, \beta}^{\rho, q}\left(\lambda(z-a)^{\alpha}\right)\right)(t) & =\sum_{k=0}^{\infty} \frac{(\rho)_{q k} \lambda^{k} \Gamma(k \alpha+\beta)(t-a)^{k \alpha+\beta-1-\mu}}{k ! \Gamma(k \alpha+\beta) \Gamma(k \alpha+\beta-\mu)} \\
& =(t-a)^{\beta-\mu-1} E_{\alpha, \beta-\mu}^{\rho, q}\left(\lambda(t-a)^{\alpha}\right) .
\end{aligned}
$$

Similarly, formulas (3.6), (3.7), and (3.8) obviously hold. 
Remark 3.4 Comparing the two fractional derivatives of the generalized MLFs, their results are identical except for the Mittag-Leffler function $E_{\alpha}(z)$. For $\operatorname{Re}(\beta)=1$, formula (3.8) will not hold because the left Caputo derivative of any constant equals zero, which is different from the Riemann-Liouville derivative. For $\operatorname{Re}(\beta)=1$, we cannot utilize (3.8) directly. By (1.2) we obtain

$$
\left({ }^{C} D_{a+}^{\mu} E_{\mu}\left(\lambda(z-a)^{\mu}\right)\right)(t)=\left({ }^{C} D_{a+}^{\mu} \sum_{k=0}^{\infty} \frac{\lambda^{k}(z-a)^{k \mu}}{\Gamma(k \mu+1)}\right)(t) .
$$

Since the series converges, we can use term-by-term differentiation. Applying (2.8) and (2.7) to the first constant term and the others of (3.10) yields

$$
\left({ }^{C} D_{a+}^{\mu} E_{\mu}\left(\lambda(z-a)^{\mu}\right)\right)(t)=\sum_{k=1}^{\infty} \frac{\lambda^{k}(t-a)^{(k-1) \mu}}{\Gamma((k-1) \mu+1)}=\lambda E_{\mu}\left(\lambda(t-a)^{\mu}\right) .
$$

\section{Two illustrative examples}

The following illustrative examples are given to show the compositions of the RL and Caputo derivatives with the Shukla function or the MLFs under different conditions.

Example 1 Let us evaluate and sketch the groups of

$$
\left(\mathbb{D}_{0+}^{0.5} E_{0.5,1}^{1,1}\left(10^{-0.6} z^{0.5}\right)\right)(t) \quad \text { and } \quad\left({ }^{C} D_{0+}^{0.5} E_{0.5,1}^{1,1}\left(10^{-0.6} z^{0.5}\right)\right)(t)
$$

Substituting the parameters $a=0, \mu=0.5$, and $\lambda=10^{-0.6}$ into (3.4) and (3.11), we obtain

$$
\left(\mathbb{D}_{0+}^{0.5} E_{0.5,1}^{1,1}\left(10^{-0.6} z^{0.5}\right)\right)(t)=\frac{t^{-0.5}}{\Gamma(0.5)}+10^{-0.6} E_{0.5}\left(10^{-0.6} t^{0.5}\right)
$$

and

$$
\left({ }^{C} D_{0+}^{0.5} E_{0.5,1}^{1,1}\left(10^{-0.6} z^{0.5}\right)\right)(t)=10^{-0.6} E_{0.5}\left(10^{-0.6} t^{0.5}\right) .
$$

The curves of the primitive function $y 1$, the RL derived function $y r$, the RL derivative of $y 1$, the Caputo derived function $y c$, the Caputo derivative of $y 1$, and the function $\frac{t^{-0.5}}{\Gamma(0.5)}$ are shown in Fig. 1.

Remark 4.1 For the condition $\beta=1$, firstly, we can see that the evaluating results of the derived unction $y r$ and the derived function $y c$ agree well with the numerical results for the RL derivative of $y 1$ and the Caputo derivative of $y 1$, respectively. Secondly, the difference between the two derivatives of the primitive function $y 1$ is $\frac{t^{-0.5}}{\Gamma(0.5)}$, which decays rapidly over time.

Example 2 Let us evaluate and sketch the groups of

$$
\left(\mathbb{D}_{0+}^{0.5} t^{1.3} E_{0.5,2.3}^{1,1}\left(10^{-2} z^{0.5}\right)\right)(t) \quad \text { and } \quad\left({ }^{C} D_{0+}^{0.5} t^{1.3} E_{0.5,2.3}^{1,1}\left(10^{-2} z^{0.5}\right)\right)(t)
$$




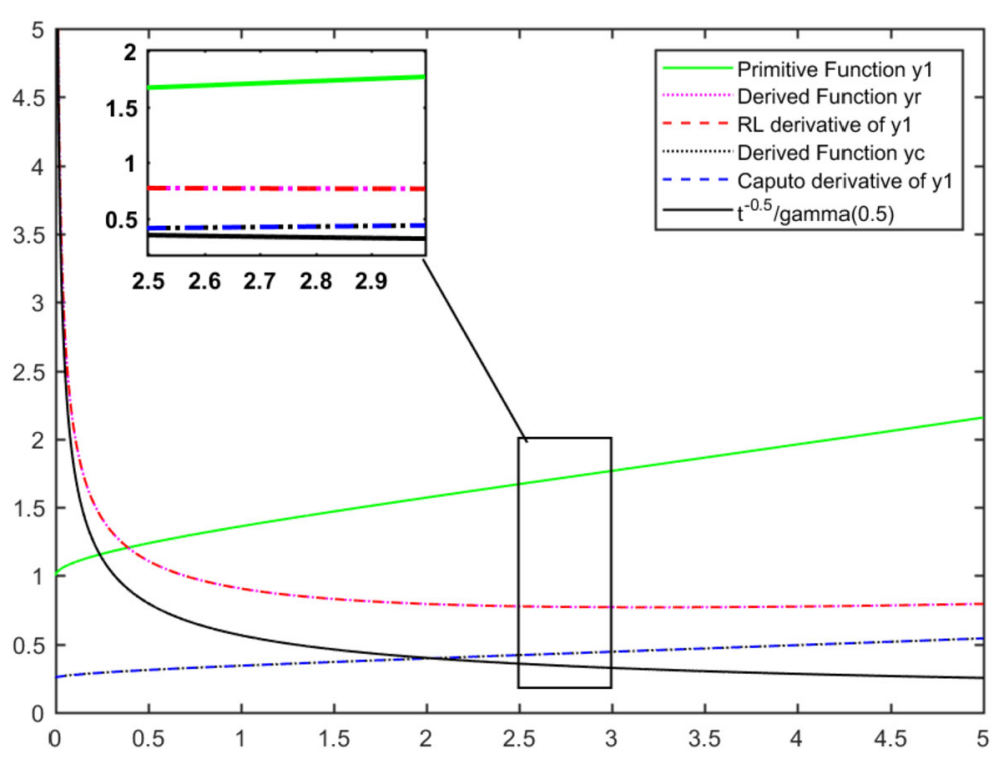

Figure 1 The fractional derivatives of the generalized MLFs for $\beta=1$

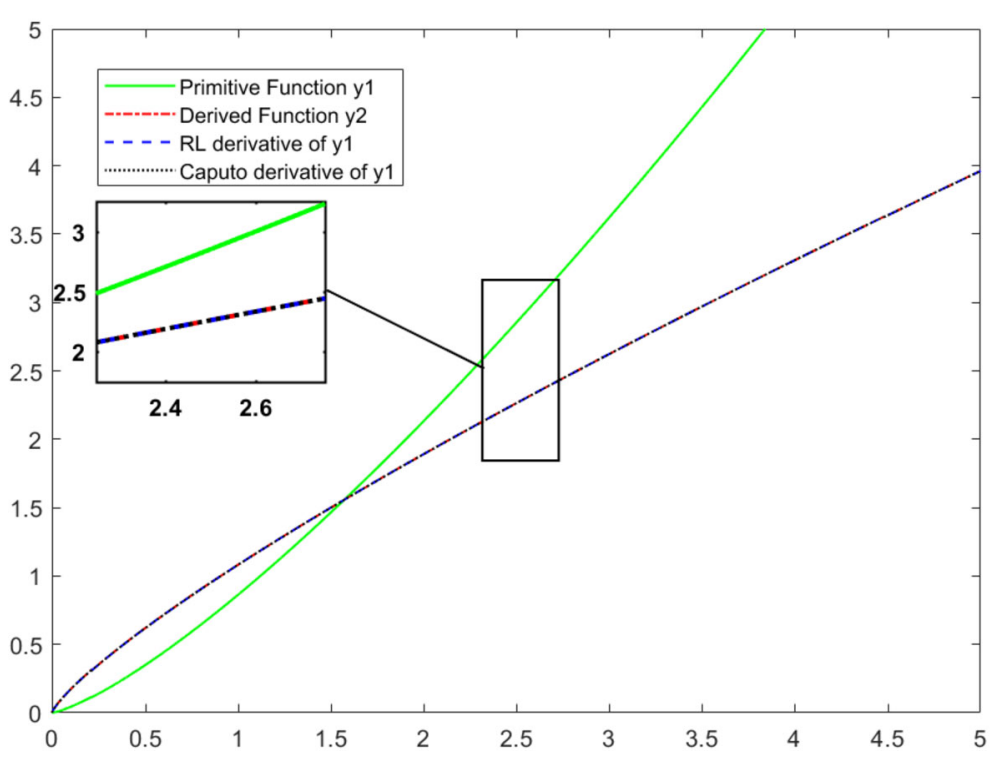

Figure 2 The fractional derivatives of the generalized MLFs for $\beta \neq 1$

Substituting the parameters $a=0, \mu=\alpha=0.5, \beta=2.3, \rho=q=1$, and $\lambda=0.01$ into (3.1) and (3.5), we get the following relations:

$$
\left(\mathbb{D}_{0+}^{0.5} t^{1.3} E_{0.5,2.3}^{1,1}\left(10^{-2} z^{0.5}\right)\right)(t)=\left({ }^{C} D_{0+}^{0.5} t^{1.3} E_{0.5,2.3}^{1,1}\left(10^{-2} z^{0.5}\right)\right)(t)=t^{0.8} E_{0.5,1.8}\left(10^{-2} t^{0.5}\right) .
$$

The comparison of the RL and Caputo derivatives with the Shukla function is shown in Fig. 2. 
Remark 4.2 For the condition $\beta=2.3 \neq 1$, the evaluating results for the derived function $y 2$ and the numerical results for the RL derivative of $y 1$ and Caputo derivative of $y 1$ agree very well with each other.

\section{Conclusions}

In this paper, we presented the compositions of the fractional derivatives with the Shukla function. We obtained the reason causing the difference between the Riemann-Liouville and Caputo derivatives of the generalized MLFs and expanded the fractional derivatives of the generalized MLFs. Finally, we provided two numerical examples to demonstrate the validity. For further research, it is interesting and challenging to discuss the relations of the new fractional derivatives and exponential or Mittag-Leffler laws [22, 32] and investigate the delayed Mittag-Leffler-type matrix functions [33] and apply them to fractional-order equations.

\section{Acknowledgements}

We sincerely appreciate the anonymous referees for their carefully reading and valuable suggestions to improve the paper.

\section{Funding}

This work was supported by National Natural Science Foundation of China (nos. 11371027, 11471015, and 11601003) and Natural Science Fund of Colleges and Universities in Anhui Province (no. KJ2018A0470).

\section{Competing interests}

The authors declare that they have no competing interests.

\section{Authors' contributions}

Each of the authors contributed equally to each part of this work. All authors read and approved the final manuscript.

\section{Publisher's Note}

Springer Nature remains neutral with regard to jurisdictional claims in published maps and institutional affiliations.

Received: 6 August 2018 Accepted: 17 October 2018 Published online: 09 November 2018

\section{References}

1. Shukla, A.K., Prajapati, J.C.: On a generalization of Mittag-Leffler function and its properties. J. Math. Anal. Appl. 336(1), 797-811 (2007)

2. Mittag-Leffler, G.: Sur la nouvelle fonction $E_{\alpha}(x)$. C. R. Acad. Sci. Paris 137, 554-558 (1903)

3. Wiman, A.: Über den fundamental Satz in der Theorie der Funktionen $E_{\alpha}(x)$. Acta Math. 29, 191-201 (1905)

4. Hille, E., Tamarkin, J.D.: On the theory of linear integral equations. Ann. Math. 31, 479-528 (1930)

5. Gross, B.: On creep and relaxation. J. Appl. Phys. 18, 212-221 (1947)

6. Barret, J.H.: Differential equations of non-integer order. Can. J. Math. 6, 529-541 (1954)

7. Caputo, M., Mainardi, F.: Linear models of dissipation in anelastic solids. Riv. Nuovo Cimento 1(2), 161-198 (1971)

8. Prabhakar, T.R.: A singular integral equation with a generalized Mittag-Leffler function in the kernel. Yokohama Math. J. 19, 7-15 (1971)

9. Kilbas, A.A., Saigo, M.: On solution of integral equations of Abel-Volterra type. Differ. Integral Equ. 8, 993-1011 (1995)

10. Sedletskii, A.M.: Asymptotic formulas for zeros of a function of Mittag-Lefler type (Russian). Anal. Math. 20, 117-132 (1994)

11. Schneider, W.R.: Complete monotone generalized Mittag-Lefler functions. Expo. Math. 14, 3-16 (1996)

12. Witte, G.: Die analytische und die numerische Behandlung einer Klasse von Volterraschen Integralgleichungen im Hilbertraum. Ph.D. Thesis, Free University of Berlin, Logos, Berlin (1997)

13. Podlubny, l.: Fractional Differential Equations. Academic Press, New York (1999)

14. Kilbas, A.A., Srivastava, H.M., Trujillo, J.J.: Theory and Applications of Fractional Differential Equations. Elsevier, Amsterdam (2006)

15. Haubold, H.J., Mathai, A.M., Saxena, R.K.: Mittag-Leffler functions and their applications. J. Appl. Math. 2011, 1-51 (2011)

16. Gorenflo, R., Kilbas, A.A., Mainardi, F., Rogosin, S.V.: Mittag-Leffler Functions Related Topics and Applications. Springer Monographs in Mathematics. Springer, Berlin (2014)

17. Kilbas, A.A., Saigo, M., Saxena, R.K.: Generalized Mittag-Leffler function and generalized fractional calculus operators. Integral Transforms Spec. Funct. 15, 31-49 (2004)

18. Li, Y., Chen, Y.Q., Podlubny, I.: Mittag-Leffler stability of fractional order nonlinear dynamic systems. Automatica 45(8), 1965-1969 (2009)

19. Monje, C.A., Chen, Y.Q., Vinagre, B.M., Xue, D.Y., Feliu, V.: In: Fractional-Order Systems and Controls: Fundamentals and Applications, London: Springer-Verlag London (2010) 
20. Zhou, Y.: Basic Theory of Fractional Differential Equations. World Scientific, Singapore (2014)

21. Wang, J.R., Zhou, Y., Lin, Z.: On a new class of impulsive fractional differential equations. Appl. Math. Comput. 242, 649-657 (2014)

22. Aguilar, J.F.G., Martínez, H.Y., Jiménez, R.F.E., Zaragoza, C.M.A., Reyes, J.R.: Analytical and numerical solutions of electrical circuits described by fractional derivatives. Appl. Math. Model. 40, 9079-9094 (2016)

23. Oliveira, D., Oliveira, E., Deif, S.: On a sum with a three-parameter Mittag-Leffler function. Integral Transforms Spec. Funct. 27(8), 639-652 (2016)

24. Aguilar, J.F.G., Jiménez, R.F.E., Lopez, M.G.L., Martínez, V.M.A., Fraga, T.C.: Electromagnetic waves in conducting media described by a fractional derivative with non-singular kernel. J. Electromagn. Waves Appl. 30(11), 1493-1503 (2016). https://doi.org/10.1080/09205071.2016.1204252

25. Ortigueira, M.D., Lopes, A.M., Machado, J.: On the computation of the multidimensional Mittag-Leffler function. Commun. Nonlinear Sci. Numer. Simul. 53, 278-287 (2017)

26. Wang, J.R., Fečkan, M., Zhou, Y.: Fractional order differential switched systems with coupled nonlocal initial and impulsive conditions. Bull. Sci. Math. 141, 727-746 (2017)

27. Barro, B.C., Hernández, M.A.T., Aguilar, J.F.G.: Application of fractional derivative with exponential law to bi-fractional-order wave equation with frictional memory kernel. Eur. Phys. J. Plus 132(515), 1-20 (2017). https://doi.org/10.1140/epjp/i2017-11796-9

28. Wang, J.R., Zhou, Y., O'Regan, D.: A note on asymptotic behaviour of Mittag-Leffler functions. Integral Transforms Spec. Funct. 29(2), 81-94 (2018)

29. Górska, K., Lattanzi, A., Dattoli, G.: Mittag-Leffler function and fractional differential equations. Fract. Calc. Appl. Anal. $21(1), 220-236(2018)$

30. Atangana, A., Aguilar, J.F.G.: Decolonisation of fractional calculus rules: breaking commutativity and associativity to capture more natural phenomena. Eur. Phys. J. Plus 133(166), 1-22 (2018). https://doi.org/10.1140/epjp/i2018-12021-3

31. Delgado, V.F.M., Aguilar, J.F.G., Hernández, M.A.T., Baleanu, D.: Modeling the fractional non-linear Schrödinger equation via Liouville-Caputo fractional derivative. Optik 162, 1-7 (2018)

32. Atangana, A.: Non validity of index law in fractional calculus: a fractional differential operator with Markovian and non-Markovian properties. Physica A 505, 688-706 (2018)

33. Li, M.M., Wang, J.R.: Exploring delayed Mittag-Leffler type matrix functions to study finite time stability of fractional delay differential equations. Appl. Math. Comput. 324, 254-265 (2018)

\section{Submit your manuscript to a SpringerOpen ${ }^{\circ}$ journal and benefit from:}

- Convenient online submission

- Rigorous peer review

- Open access: articles freely available online

- High visibility within the field

- Retaining the copyright to your article

Submit your next manuscript at $\boldsymbol{~ s p r i n g e r o p e n . c o m ~}$ 